\title{
INTERFERON REGULATORY FACTOR 7- (IRF7-) MEDIATED IMMUNE RESPONSE AFFECTS NEWCASTLE DISEASE VIRUS REPLICATION IN CHICKEN EMBRYO FIBROBLASTS
}

\author{
Zhaoxiong $\mathrm{WANG}^{1,2+}$, Zhangyong $\mathrm{NING}^{1+}$, Minhua $\mathrm{SuN}^{1}$, Shimin $\mathrm{GAO}^{1}$, Yinfeng KANG ${ }^{1}$, \\ Peng XIE ${ }^{1}$ and Tao REN ${ }^{1 *}$ \\ ${ }^{1}$ Key Laboratory of Animal Diseases Control and Prevention of the Ministry of Agricul- \\ ture, College of Veterinary Medicine, South China Agricultural University, $483 \mathrm{Wu}$ Shan \\ Road, Tian He District, Guangzhou 510642, P. R. China; ${ }^{2}$ College of Animal Science, \\ Yangtze University, Jingzhou, Hubei, P. R. China
}

(Received 17 September 2013; accepted 25 June 2014)

\begin{abstract}
Interferon regulatory factor 7 (IRF7) is essential for the induction of an antiviral response. Previous studies have shown that virus replication causes the activation or expression of Type I interferon (IFN) in cells, which further activates IFN-stimulated genes (ISGs) to retard virus growth. In this study, after infection of chicken embryo fibroblasts (CEFs) with the lentogenic Newcastle disease virus (NDV) strain LaSota or the velogenic NDV strain GM, the mRNA and protein levels of IRF7 showed a significant increase, and part of the IRF7 protein was translocated from the cytoplasm to the nucleus. In order to further explore the effect of IRF7-mediated innate immune response on the replication of NDV in CEFs, the mRNA levels of IFN- $\alpha$, IFN- $\beta$ and STAT1 were measured and the replication kinetics of NDV determined. The results showed that specific siRNA could inhibit the expression of IRF7 and limit the mRNA level of IFN- $\alpha$, IFN- $\beta$ and STAT1 and, accordingly, the replication kinetics of both NDVs were enhanced after the inhibition of IRF7. In conclusion, IRF7 is an important nuclear transcription factor for the induction of Type I IFNs during the antiviral response, which can affect the replication of NDV and spread to CEFs in the early phase of viral infection.
\end{abstract}

Key words: Newcastle disease virus, IRF7, interferon, virus replication, chicken embryo fibroblast

Viral infection elicits the activation of numerous cellular signal transduction pathways, leading to the induction of both innate and adaptive immune responses to limit further dissemination of the virus throughout the host. Pattern recognition receptors (PRRs), including transmembrane toll-like receptors (TLRs) or cytosolic sensors (RIG-I, MDA5, PKR and NOD proteins), are recognised by

${ }^{+}$Co-first authors; ${ }^{*}$ Corresponding author; E-mail: rentao6868@126.com; Phone: 0086 (20) 8528-3054; Fax: 0086 (20) 8528-0242 
viral components, leading to IFN-mediated and inflammatory responses (Sarkar et al., 2004; Yoneyama et al., 2005; Kato et al., 2006; Liu et al., 2007; Loo et al., 2008). In recent years, extensive studies of the interferon regulatory factor (IRF) family have confirmed that IRFs have gained great attention due to their diverse roles such as initiating antiviral responses, regulating inflammatory cytokine expression and controlling cell cycle and apoptosis, as well as mediating the development of macrophages, dendritic cells, B and T lymphocytes (Au et al., 1998; Sato et al., 1998; Honda et al., 2005; Ning et al., 2011). As an important transcription mediator, IRF7 has been initially identified to induce the expression of IFNs and IFN-stimulated genes (ISGs) in the hosts, and it plays a critical role in initiating host innate immune responses to viral and bacterial infection (Marie et al., 1998; Smith et al., 2001).

Newcastle disease (ND) caused by Newcastle disease virus (NDV), a member of the Paramyxoviridae family, is recognised as a dangerous disease of poultry, which leads to severe economic losses to the poultry industry all over the world. NDV isolates are usually classified into three groups, i.e. avirulent (lentogenic), mildly virulent (mesogenic) and highly virulent (velogenic), on the basis of their virulence. Virulent NDV elicits a strong innate immune response in chickens (Rue et al., 2011), but the knowledge existing on the role of IRF7 in that response and on the effect of IRF7 on NDV replication is still limited. In the present study, we investigated the expression of IRF7, IFN- $\alpha$, IFN- $\beta$ and STAT1, detected the distribution of IRF7 protein in NDV-infected chicken embryo fibroblasts (CEFs), and determined the kinetics of NDV replication after the inhibition of IRF7. The experimental results indicated that two NDV strains of different virulence could positively regulate the IRF7-mediated signalling pathway, increase the expression of IFN- $\alpha$ and IFN- $\beta$, and limit the replication of NDVs.

\section{Materials and methods}

\section{Cells}

Chicken embryo fibroblast cells (CEFs) and Vero cells (African green monkey kidney epithelial cells, ATCC) were maintained in D-Minimum Essential Medium (D-MEM, Gibco ${ }^{\circledR}$ ) supplemented with $10 \%$ fetal bovine serum (FBS; PAA Laboratories, Ontario, Canada), respectively.

\section{Viruses}

The lentogenic NDV strain LaSota and the velogenic NDV strain GM (genotype VII) were preserved in our laboratory. The GenBank accession numbers of LaSota and GM strains are AF077761 and DQ486859, respectively. The virus titres were determined on CEF and Vero cells. UV inactivation of viruses was performed by the use of a UV Stratalinker 2400 (Stratagene, USA). 
Real-time quantitative polymerase chain reaction ( $q P C R$ )

The CEFs were infected with NDV strain LaSota or GM at a multiplicity of infection (MOI) of 0.07 in basal D-MEM. The cells were harvested at 3, 6, 12 and $24 \mathrm{~h}$ post infection (p.i.). Real-time PCR analysis for mRNA levels of IRF7, IFN- $\alpha$, IFN- $\beta$ and STAT1 genes were normalised using the glyceraldehyde-3phosphate dehydrogenase (GAPDH) gene as internal standard (Table 1). The Tm and $\mathrm{Cp}$ values were calculated by using the analysis software. Fold changes in the expression of each gene were compared with the expression in non-infected samples, and were analysed by a comparative threshold cycle $(\mathrm{Ct})$ method using the formula of $2^{-(\Delta \Delta \mathrm{Ct})}$.

Table 1

Primer sequences and the size of amplicons generated by SYBR Green based real-time PCR

\begin{tabular}{|c|c|c|c|}
\hline Primer & Sequence $\left(5^{\prime}-3^{\prime}\right)$ & $\begin{array}{l}\text { Amplicon size } \\
\text { (bp) }\end{array}$ & $\begin{array}{l}\text { Target gene } \\
\text { (GenBank } \\
\text { accession no.) }\end{array}$ \\
\hline $\begin{array}{l}\text { IFN- } \alpha-S \\
\text { IFN- } \alpha-A S\end{array}$ & $\begin{array}{l}\text { AAGCCGACAAAACCACCC } \\
\text { CAGGAACCAGGCACGAGC }\end{array}$ & 302 & AM049251 \\
\hline $\begin{array}{l}\text { IFN- } \beta-S \\
\text { IFN- } \beta \text {-AS }\end{array}$ & $\begin{array}{l}\text { CCTCAACCAGATCCAGCATT } \\
\text { GGATGAGGCTGTGAGAGGAG }\end{array}$ & 259 & X92479 \\
\hline $\begin{array}{l}\text { IRF7-S } \\
\text { IRF7-AS }\end{array}$ & $\begin{array}{l}\text { AAGCCCAAGGAGTCCAAGCTC } \\
\text { TCAGAGAGCTGCAGGCTGAC }\end{array}$ & 147 & NM_205372 \\
\hline $\begin{array}{l}\text { STAT1-S } \\
\text { STAT1-AS }\end{array}$ & $\begin{array}{l}\text { GAAACGGCTACATTAGGACTG } \\
\text { GATCCGAGATACCTCATCAAAC }\end{array}$ & 119 & NM_001012914 \\
\hline $\begin{array}{l}\text { GAPDH-S } \\
\text { GAPDH-AS }\end{array}$ & $\begin{array}{l}\text { ССТCTCTGGCAAAGTCCAAG } \\
\text { CATCTGCCCATTTGATGTTG }\end{array}$ & 200 & V00407 \\
\hline
\end{tabular}

$\mathrm{A}=$ adenine, $\mathrm{G}=$ guanosine, $\mathrm{C}=$ cytosine, $\mathrm{T}=$ thymidine

\section{SDS-PAGE and Western blotting}

Whole-cell extracts were prepared as described previously (Noyce et al., 2006). Proteins were separated by sodium dodecyl sulphate-polyacrylamide gel electrophoresis (SDS-PAGE) and transferred onto nitrocellulose (NC) membranes. The membranes were incubated with primary rabbit anti-IRF7 antibody (Epitomics, USA) that can detect IRF7 from chicken. Then the NC membranes were incubated with FITC-conjugated goat anti-rabbit antibody (Millipore, Billerica, USA). Following the incubation with secondary antibody, protein bands were imaged with the Odyssey infrared imaging system (Li-Cor, Odyssey). The GAPDH expression level measured in parallel served as control. 


\section{Immunofluorescence staining}

CEFs were infected with NDV at a MOI of 0.07 or were treated with NDVUV at the same titre. The cells were fixed with $4 \%$ paraformaldehyde. After incubation with primary rabbit anti-IRF7 antibody (Epitomics, USA), the cells were stained with FITC-conjugated goat anti-rabbit secondary antibody (Millipore, Billerica, USA). In order to stain the nuclei, 4',6-diamidino-2-phenylindole (DAPI; Sigma) was added to incubation medium with the cells. The stained cells were examined under a laser confocal microscope (Olympus, Japan).

\section{IRF7 siRNA treatment}

The specific siRNA (GenePharma, China) for chicken IRF7 (5'-CAACCG CAAAGAAGGGUUGCGUUUA-3' and 5'-UAAACGCAACCCUUCUUUGCG GUUG-3') was transfected into the cells at a concentration of $40 \mathrm{pM}$. Nontargeting siRNA (sense strand: 5'-UUCUCCGAACGUGUCACGUTT-3', antisense strand: 5'-ACGUGACACGUUCGGAGAATT-3') was used as control. Inhibitory efficiency was monitored using qPCR analysis for IRF7, IFN- $\alpha$, IFN- $\beta$ and STAT1. Fold changes in the expression of each gene were compared with its expression level in normal cells.

\section{Growth kinetics of NDV}

A total of $40 \mathrm{pM}$ IRF7 siRNA was transfected into cells at $70 \%$ confluence. After $24 \mathrm{~h}$ incubation, the cell monolayers were infected with NDV strain LaSota at a MOI of 0.7 or with strain GM at a MOI of 0.07 , respectively, and then incubated at $37{ }^{\circ} \mathrm{C}$ in virus-producing medium. Supernatants were harvested every $12 \mathrm{~h}$, and virus titres were determined on Vero cells.

\section{Statistical analysis}

The statistical significance of the experimental data was analysed by Student's $t$-test.

\section{Results}

NDV upregulates mRNA levels of IRF7, IFN- $\alpha$, IFN- $\beta$ and STAT1. As shown in Fig. 1, two NDV strains of different virulence could differentially modulate the mRNA levels of four genes. After NDV LaSota infection for $3 \mathrm{~h}$ in CEFs, the mRNA levels of IRF7, IFN- $\alpha$, IFN- $\beta$ and STAT1 revealed a 92-, 8-, 19- and 23-fold increase, respectively, when compared with those in the normal control cells. Meanwhile, the four genes revealed a 12-, 2.8-, 1.4- and 1.5-fold increase after infection with the velogenic NDV strain GM. With the extension of infection time, the mRNA levels of the four genes exhibited an obvious 
downward trend. Taken together, after infection of the CEFs with a velogenic or lentogenic NDV strain, the mRNA levels of IFN- $\alpha$, IFN- $\beta$ and STAT1 were upregulated; however, there was an obvious difference in mRNA induction of the four genes between the two NDV strains.
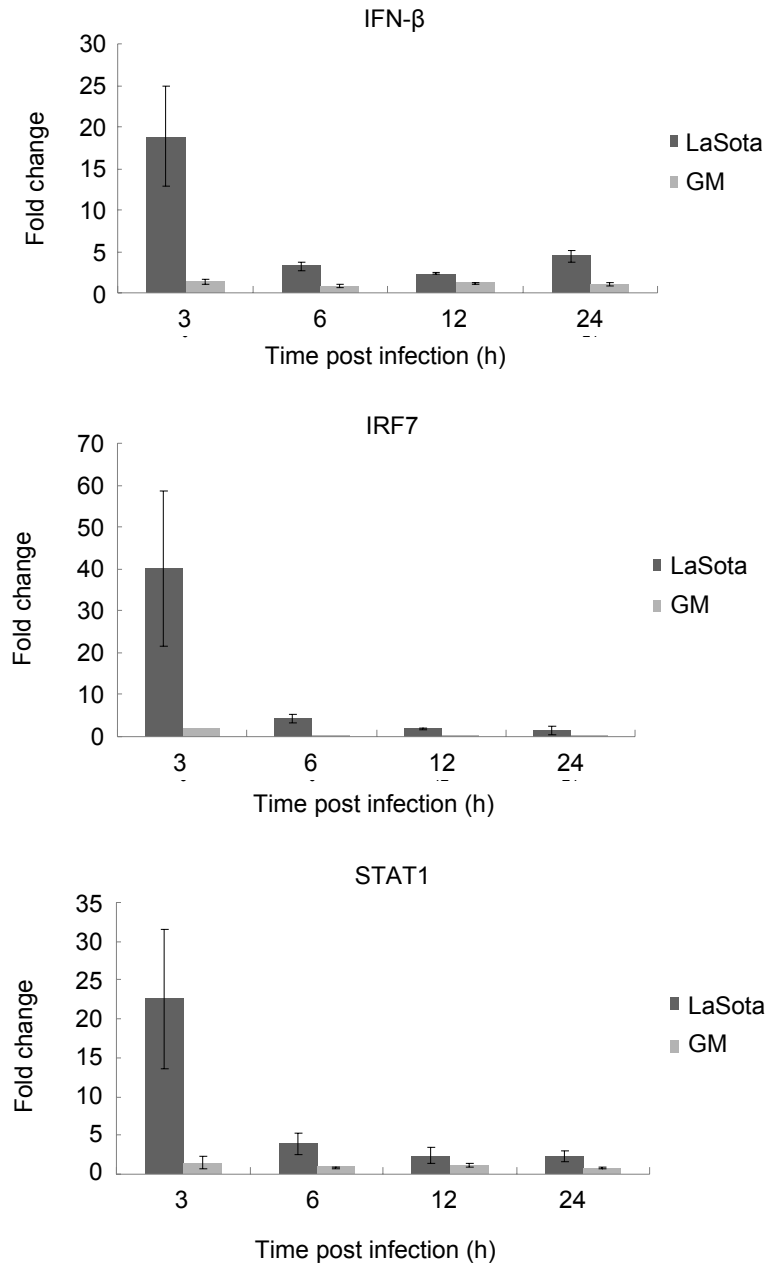

Fig. 1. Induction of IRF7, IFN- $\alpha$, IFN- $\beta$ and STAT1 mRNA in CEFs (A). After the infection of CEFs with NDV LaSota or GM, the cells were harvested at 3, 6, 12 and $24 \mathrm{~h}$ post infection (p.i.). qPCR analysis on mRNA levels of IRF7, IFN- $\alpha$, IFN- $\beta$ and STAT1 genes was normalised by the use of GAPDH gene as internal control

NDV upregulates the expression of IRF7. Western blotting analysis showed that NDV LaSota or GM infection induced different IRF7 protein expression levels. As displayed in Fig. 2, the cells could synthesise and secrete more 
IRF7 protein in NDV-infected CEFs at a MOI of 0.07 when compared with the mock-infected group. In addition, some differences in the relative amount of IRF7 protein induced by the two NDVs were observed. In CEFs infected with velogenic NDV strain GM for $3 \mathrm{~h}$, the IRF7 protein revealed a marked increase; while CEFs synthesised more IRF7 protein in the group infected with NDV strain LaSota at a MOI of 0.07 . These results suggested that NDVs of different virulence could induce the CEFs to synthesise IRF7 protein and activated the IRF7-mediated signalling pathway. On the other hand, the difference of IRF7 protein expression in the two groups indicated that NDVs of different virulence revealed different capabilities in activating the IRF7-mediated signalling pathway.

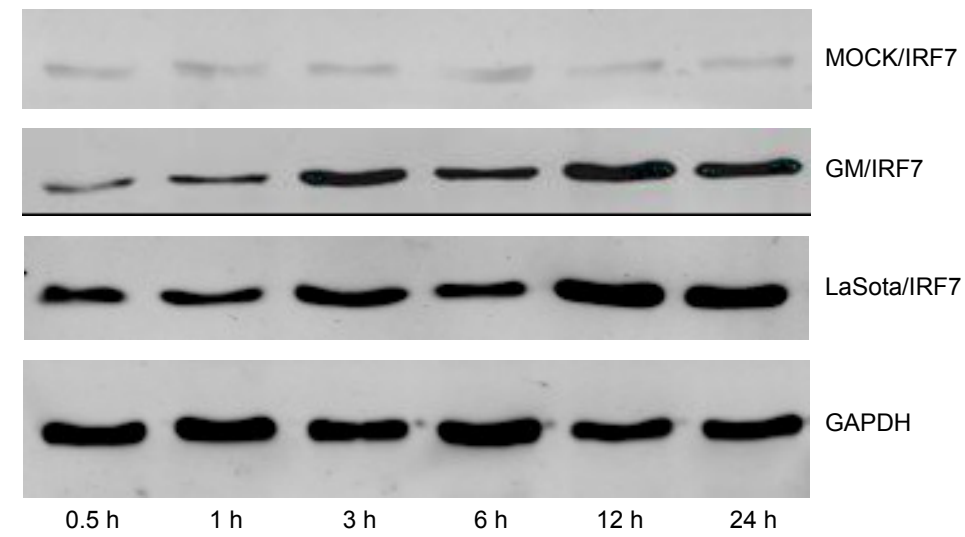

IRF7

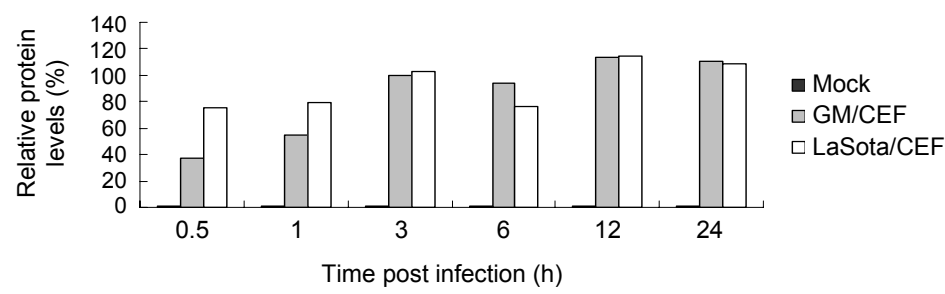

Fig. 3. Status of IRF7 protein in CEFs. CEFs were infected by activated NDV strain LaSota or GM, or were treated with UV-inactivated NDV (NDV-UV) strain LaSota or GM. Cells were fixed with a stationary liquid. They were incubated with rabbit IRF7 antibody and then stained with goat anti-rabbit antibody labelled with FITC. DAPI was added to CEFs to stain the nuclei. The cells were observed under a laser confocal microscope

NDV promotes IRF7 protein localisation in the nucleus. In the present study, most of the IRF7 translocates from the cytoplasm to the nucleus after infection by lentogenic or velogenic NDV in cells (Fig. 3, upper two panels). However, in the mock-infected CEFs, IRF7 is located predominantly in the cytoplasm (Fig. 3, middle panel). In order to gain further insights into the transloca- 
tion mechanism of IRF7, laser confocal analysis was conducted after CEFs had been treated with UV-inactivated NDV strain GM or LaSota for $24 \mathrm{~h}$. The results showed that IRF7 located predominantly in the cytoplasm (Fig. 3, lower two panels), which indicated that the activated NDV could induce the shift of IRF7 protein from the cytoplasm to the nucleus, while the inactivated virus could not exert this function in CEFs. Therefore, the nuclear translocation of IRF7 is probably correlated with virus replication.

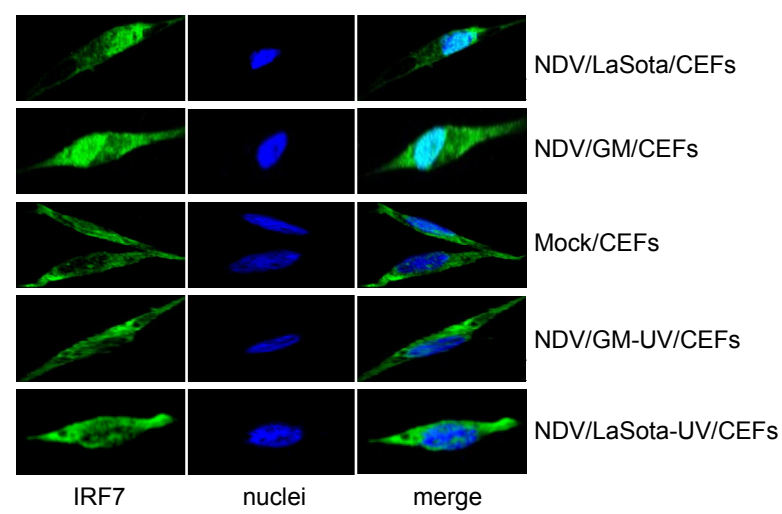

Fig. 3. Status of IRF7 protein in CEFs. CEFs were infected by activated NDV strain LaSota or GM, or were treated with UV-inactivated NDV (NDV-UV) strain LaSota or GM. Cells were fixed with a stationary liquid. They were incubated with rabbit IRF7 antibody and then stained with goat anti-rabbit antibody labelled with FITC. DAPI was added to CEFs to stain the nuclei. The cells were observed under a laser confocal microscope

Inhibition of chicken IRF7 negatively regulates IFN- $\alpha$, IFN- $\beta$ and STAT1. After IRF7 siRNA was transfected to CEFs for $24 \mathrm{~h}$, the mRNA levels of IRF7, IFN- $\alpha$, IFN- $\beta$ and STAT1 were evaluated by qPCR. Compared with the normal group, the mRNA level of IRF7 revealed a more than $70 \%$ decrease and, correspondingly, the mRNA level of IFN- $\alpha$, IFN- $\beta$ and STAT1 exhibited an approximately $50 \%$ decline (Fig. 4). Compared with the normal cells, the fold changes in mRNA levels of IRF7, IFN- $\alpha$, IFN- $\beta$ and STAT1 were not obvious after the cells had been treated with non-targeting siRNA. These results showed that the suppression of IRF7 could inhibit the mRNA level of Type 1 IFN, suggesting that the IRF7-mediated signalling pathway plays an important role in the process of antiviral response.

Inhibition of IRF7 enhances NDV replication. After the CEFs had been treated with specific siRNA for chicken IRF7 for $24 \mathrm{~h}$, and the cells had been infected with NDV strain GM or LaSota, cell supernatants were harvested in order to examine virus-producing kinetics on Vero cells. In this study, in the presence of NDV strain GM or LaSota, the virus-producing kinetics was enhanced when compared with that of the control group if IRF7 was inhibited in advance (Fig. 5). 
Therefore, the IRF7-mediated innate immune response plays an important role in NDV replication.

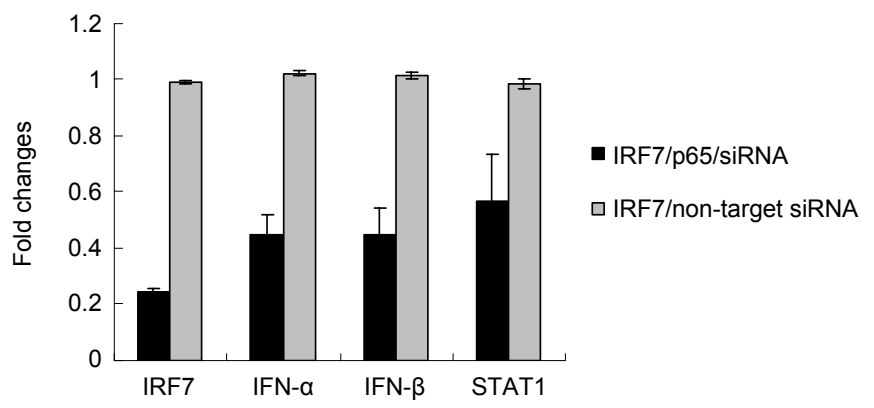

Fig. 4. Inhibition of IRF7 negatively regulates the mRNA level of IFN- $\alpha$, IFN- $\beta$ and STAT1. The cells were treated with 20 pM oligonucleotide of IRF7-non-specific siRNA as control or IRF7specific siRNA, then collected to extract the RNA, and IRF7, IFN- $\alpha$, IFN- $\beta$ and STAT1 mRNA levels were determined by qPCR

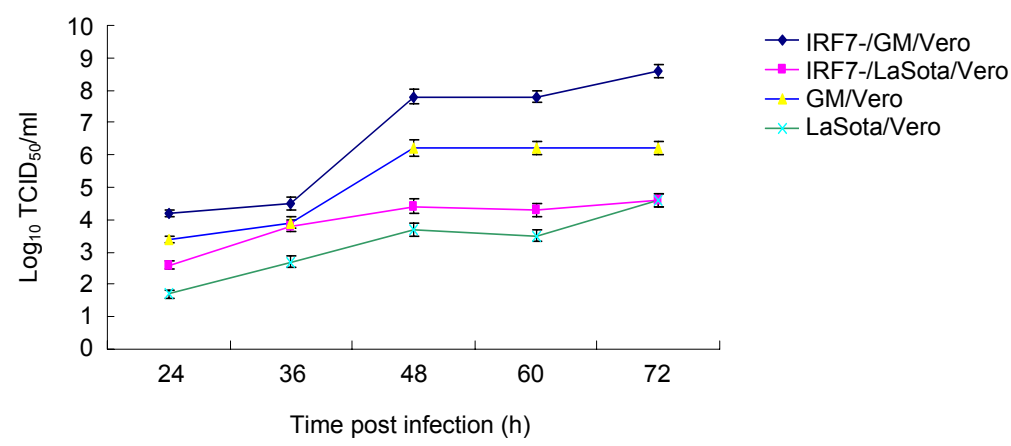

Fig. 5. NDV production kinetics between the control group and IRF7 siRNA cell lines. The cells were treated with $100 \mathrm{pM}$ oligonucleotide of IRF7-non-specific siRNA as control or IRF7-specific siRNA, and then cells were infected with NDV strain GM or LaSota. The cell supernatant was collected at 24, 36, 48, 60 and $72 \mathrm{~h}$ p.i., and virus titres were determined on Vero cells

\section{Discussion}

Interferons are important components of the innate immune response to viral infection (Borrow et al., 2010). Early response to virus causes the secretion of a subset of IFNs in the host through PRRs recognised as pathogen-associated molecular patterns (PAMPS), and further results in the activation of transcription factors such as IRF-3, IRF7, NF-kB and activator protein-1 (AP-1) (c-jun/ATF). Once Type I IFNs are synthesised, they will play a key role in the antiviral response by mediating direct upregulation of ISGs, activating natural cells (NC) and dendritic cells (DC), and promoting the induction of adaptive response to limit viral replication (Mogensen et al., 1999; Pansky et al., 2000; Sharma et al., 2003). 
Different viruses infecting hosts will cause a corresponding change of the IRF7 signalling pathway. IRF7 has been identified to play a role in the context of Epstein-Barr virus (EBV) infection, and is regarded as the crucial regulator of Type I IFN against pathogenic infections. Christensen et al. (2012) have demonstrated that early Type I IFN response to lymphocytic choriomeningitis virus (LCMV) infection in the central nervous system (CNS) is controlled by a concerted action of IRF3 and IRF7. Previous studies have shown that human plasmacytoid dendritic cells (pDCs) can express IRF7 constitutively, but this basal expression is rapidly upregulated after the stimulation and is maintained in vitro only in the presence of a stimulant. Further studies have demonstrated that nuclear translocation of IRF7 occurs earlier than IRF7 induction (Au et al., 1998; Sato et al., 1998; Honda et al., 2005; Ning et al., 2011). The characterisation of cellular components involved in viral detection and IRF activation will further delineate this vital mechanism of the innate immune response.

The IRF7-mediated innate immune response can affect the replication of different viruses. Overexpression of IRF7 can result in the enhancement of viral replication, while the knockdown of IRF7 in macrophages can significantly reduce viral production (Sirois et al., 2011). Ruddy et al. (2012) have demonstrated that Chikungunya virus (CHIKV) infection of IRF3- or IRF7-deficient adult mice is lethal, and the mortality is associated with undetectable serum IFN- $\alpha / \beta$, and with increased virus replication.

However, it is unclear how the IRF7-mediated signalling pathway changes in NDV-infected CEFs, and how the pathway change affects NDV replication in turn. Our study shows that virulent or lentogenic NDV can activate IRF7mediated signalling pathway. Compared with the control group, IRF7 mRNA and protein levels are obviously increased after infection by both NDVs in CEFs, and IFN- $\alpha$ and IFN- $\beta$ mRNA levels are increased correspondingly. Confocal laser trials have also demonstrated that IRF7 mainly distributes in the cytoplasm in the normal CEF group and in the NDV-UV virus-treated CEF group, while the IRF protein is mainly distributed in the nucleus after a clear translocation from the cytoplasm following infection by NDVs in CEFs.

Lentogenic and velogenic NDVs have different capabilities to induce the expression of IFN- $\alpha$, IFN- $\beta$ and STAT1 in CEFs. An important factor responsible for this is probably the $\mathrm{V}$ protein of NDV that functions as an IFN- $\alpha / \beta$ antagonist targeting STAT1 (Huang et al., 2003; Park et al., 2003; Nishio et al., 2005). The difference between $\mathrm{V}$ proteins derived from velogenic NDV-GM and lentogenic NDV-LaSota may be their different ability to target STAT1, which is worthy of further study in the future. Another possible cause may be correlated with apoptosis. NDV is known to cause apoptosis in different cell types including CEFs, Vero cells and peripheral blood mononuclear cells. Therefore, the entry and replication of viruses should be required during NDV-induced apoptosis (Lam, 
1995, 1996; Geisler et al., 2007), and different apoptosis rates induced by lentogenic and velogenic NDVs may lead to differences in protein production in CEFs.

Subsequent studies have explored the effect of the IRF7-mediated signalling pathway on NDV replication by the inhibition of IRF7 siRNA. In the present study, the mRNA levels of IFN- $\alpha$ and IFN- $\beta$ were significantly decreased and the virus titre showed a clear and significant increase after virus infection in CEFs, indicating that the IRF7-mediated signalling pathway can suppress the replication of NDV.

It is important to choose an appropriate cell line to support the efficient production of NDV. As primary cells, CEFs are more suitable for studying antiviral response than other cell lines. In this study, we have found that the entry of NDVs into CEFs can result in the activation IRF7, and virus-induced activation of IFN- $\alpha$ and IFN- $\beta$ genes will be mediated by IRF7. Once IFN- $\alpha$ and IFN- $\beta$ proteins are expressed in CEFs, these proteins can execute the innate immune response to retard the replication of NDVs by activating the Janus kinase/signal transducer and activator of transcription (JAK/STAT) signal transduction cascade in both autocrine and paracrine manners, thus leading to the activation of ISGs to delay the further replication of NDVs. After inhibition of IRF7 by specific siRNA, the mRNA level of IFN- $\alpha / \beta$ and STAT will decline significantly in CEFs and, accordingly, the virus-producing kinetics will exhibit a remarkable enhancement.

It can be concluded that the IRF7-mediated signalling pathway can be activated after CEFs are infected with NDV, and the change in the signalling pathway can affect the replication of NDVs. These investigations can provide an insight into the mechanism of IRF7-mediated innate immune response elicited by NDV infection and the response to viral replication in the host.

\section{Acknowledgements}

This work was supported by a grant from the National Natural Science Foundation of China $(31072319,31372412)$ and the Specialized Research Fund for the Doctoral Program of Higher Education of China (20124404110016).

\section{References}

Au, W. C., Moore, P. A., LaFleur, D. W., Tombal, B. and Pitha, P. M. (1998): Characterization of the interferon regulatory factor-7 and its potential role in the transcription activation of interferon A genes. J. Biol. Chem. 273, 29210-29217.

Borrow, P., Sobrido, L. M. and de la Torre, J. C. (2010): Inhibition of the Type I interferon antiviral response during arenavirus infection. Viruses 2, 2443-2480.

Christensen, J. E., Fenger, C., Issazadeh-Navikas, S., Krug, A, Liljestrøm, P., Goriely, S., Paludan, S. R., Finsen, B., Christensen, J. P. and Thomsen, A. R. (2012): Differential impact of in- 
terferon regulatory factor 7 in initiation of the type I interferon response in the lymphocytic choriomeningitis virus-infected central nervous system versus the periphery. J. Virol. 86, 7384-7392.

Geisler, F., Algül, H., Paxian, S. and Schmid, R. M. (2007): Genetic inactivation of RelA/p65 sensitizes adult mouse hepatocytes to TNF-induced apoptosis in vivo and in vitro. Gastroenterology 132, 2489-2503.

Honda, K., Yanai, H., Negishi, H., Asagiri, M., Sato, M., Mizutani, T., Shimada, N., Ohba, Y., Takaoka, A., Yoshida, N. and Taniguchi, T. (2005): IRF-7 is the master regulator of type-I interferon-dependent immune responses. Nature 434, 772-777.

Huang, Z., Krishnamurthy, S., Panda, A. and Samal, S. K. (2003): Newcastle disease virus V protein is associated with viral pathogenesis and functions as an alpha interferon antagonist. J. Virol. 77, 8676-8685.

Kato, H., Takeuchi, O. and Sato, S. (2006): Differential roles of MDA5 and RIG-I helicases in the recognition of RNA viruses. Nature 441, 101-105.

Lam, K. M. (1995): Apoptosis in chicken embryo fibroblasts caused by Newcastle disease virus. Vet. Microbiol. 47, 357-363.

Lam, K. M. (1996): Newcastle disease virus-induced apoptosis in peripheral blood mononuclear cells of chickens. J. Comp. Pathol. 114, 63-71.

Liu, P, Jamaluddin, M., Li, K., Garofalo, R. P., Casola, A. and Brasier, A. R. (2007): Retinoic acid-inducible gene I mediates early antiviral response and Toll-like receptor 3 expression in respiratory syncytial virus-infected airway epithelial cells. J. Virol. 81, 1401-1411.

Loo, Y. M., Fornek, J., Crochet, N., Bajwa, G., Perwitasari, O., Martinez-Sobrido, L., Akira, S., Gill, M. A. and García-Sastre, A., Katze, M. G. and Gale, M. Jr. (2008): Distinct RIG-I and MDA5 signaling by RNA viruses in innate immunity. J. Virol. 82, 335-345.

Marie, I., Durbin, J. E. and Levy, D. E. (1998): Differential viral induction of distinct interferonalpha genes by positive feedback through interferon regulatory factor-7. EMBO J. 17, 6660-6669.

Mogensen, K. E., Lewerenz, M., Reboul, J., Lutfalla, G. and Uzé, G. (1999): The type I interferon receptor: structure, function, and evolution of a family business. J. Interferon Cytokine Res. 19, 1069-1098.

Ning, S., Pagano, J. S. and Barber, G. N. (2011): IRF7: activation, regulation, modification and function. Genes Immu. 12, 399-414.

Nishio, M., Tsurudome, M., Ito, M., Garcin, D., Kolakofsky, D. and Ito, Y. (2005): Identification of paramyxovirus $\mathrm{V}$ protein residues essential for STAT protein degradation and promotion of virus replication. J. Virol. 79, 8591-8601.

Noyce, R. S., Collins, S. E. and Mossman, K. L. (2006): Identification of a novel pathway essential for the immediate-early, interferon-dependent antiviral response to enveloped virions. J. Virol. 80, 226-235.

Pansky, A., Hildebrand, P., Fasler-Kan, E., Baselgia, L., Ketterer, S., Beglinger, C. and Heim, M. H. (2000): Defective Jak-STAT signal transduction pathway in melanoma cells resistant to growth inhibition by interferon-alpha. Int. J. Cancer 85, 720-725.

Park, M. S., García-Sastre, A., Cros, J. F., Basler, C. F. and Palese, P. (2003): Newcastle disease virus $\mathrm{V}$ protein is a determinant of host range restriction. J. Virol. 77, 9522-9532.

Ruddy, P. A., Wilson, J., Gardner, J., Larcher, T., Babarit, C., Le, T. T., Anraku, I., Kumagai, Y., Loo, Y. M., Gale, M. J., Akira, S., Khromykh, A. A. and Suhrbier, A. (2012): Interferon response factors 3 and 7 protect against Chikungunya virus hemorrhagic fever and shock. J. Virol. 86, 9888-9898.

Rue, C. A., Susta, L., Cornax, I., Brown, C. C., Kapczynski, D. R., Suarez, D. L., Miller, P. J. and Afonso, C. L. (2011): Virulent Newcastle disease virus elicits a strong innate immune response in chickens. J. Gen. Virol. 92, 931-939. 
Sarkar, S. N., Peters, K. L., Elco, C. P., Sakamoto, S., Pal, S. and Sen, G. C. (2004): Novel roles of TLR3 tyrosine phosphorylation and PI3 kinase in double-stranded RNA signaling. Nat. Struct. Mol. Biol. 11, 1060-1067.

Sato, M., Hata, N., Asagiri, M., Nakaya, T., Taniguchi, T. and Tanaka, N. (1998): Positive feedback regulation of type I IFN genes by the IFN-inducible transcription factor IRF7. FEBS Lett. 441, 106-110.

Sharma, S., tenOever, B. R., Grandvaux, N., Zhou, G. P., Lin, R. and Hiscott, J. (2003): Triggering the interferon antiviral response through an IKK-related pathway. Science 300, 1148-1151.

Sirois, M., Robitaille, L., Allary, R., Shah, M., Woelk, C. H., Estaquier, J. and Corbeil, J. (2011): TRAF6 and IRF7 control HIV replication in macrophages. PLoS One 6 (11): e28125.

Smith, E. J., Marie, I., Prakash, A., Garcia-Sastre, A. and Levy, D. E. (2001): IRF3 and IRF7 phosphorylation in virus-infected cells does not require double-stranded RNA-dependent protein kinase R or Ikappa B kinase but is blocked by vaccinia virus E3L protein. J. Biol. Chem. 276, 8951-8957.

Yoneyama, M., Kikuchi, M., Matsumoto, K., Imaizumi, T., Miyagishi, M., Taira, K., Foy, E., Loo, Y. M., Gale, M. Jr., Akira, S., Yonehara, S., Kato, A. and Fujita, T. (2005): Shared and unique functions of the DExD/H-box helicases RIG-I, MDA5, and LGP2 in antiviral innate immunity. J. Immunol. 175, 2851-2858. 